\title{
Comunicação interprofissional em unidade de emergência: estudo de caso*
}

\author{
Interprofessional communication in an emergency care unit: a case study \\ Comunicación interprofesional en Unidad de Urgencias Hospitalarias: estudio de caso
}

Como citar este artigo:

Coifman AHM, Pedreira LC, Jesus APS, Batista REA. Interprofessional communication in an emergency care unit: a case study. Rev Esc Enferm USP. 2021;55:e03781. https://doi.org/10.1590/S1980-220X2020047303781

\section{Dlyne Henri Motta Coifman ${ }^{1}$ \\ Larissa Chaves Pedreira ${ }^{1}$ \\ Ana Paula Santos de Jesus ${ }^{2}$ \\ Ruth Ester Assayag Batista ${ }^{3}$}

* Extraído da tese "Produção de sentido da comunicação interprofissional para profissionais de saúde na Unidade de Emergência: estudo de caso", Universidade Federal da Bahia, 2019.

${ }^{1}$ Universidade Federal da Bahia, Salvador, BA, Brasil.

${ }^{2}$ Universidade Federal do Recôncavo da Bahia, Santo Antônio de Jesus, BA, Brasil.

${ }^{3}$ Universidade Federal de São

Paulo, São Paulo, SP, Brasil.

\begin{abstract}
Objective: To map internal and external factors in an emergency care unit that interfere with interprofessional communicative practice. Method: This is a single case study carried out in the emergency care unit of a general hospital. Data were collected through participant observation, document analysis, and semi-structured interviews, and were triangulated and subjected to thematic analysis, out of categories defined a priori, based on the SWOT matrix. Results: Twenty-two health care professionals participated in the study. As for the strengths and opportunities, it was found that professionals understand the importance of communication as a safety measure, and they use the shift change and written communication to share information. However, overcrowding, work overload, the lack of behaviors standardization, the inexperience of professionals, and the deficit in the interprofessional relationship are factors that hinder effective communication. Conclusion: The fragile interprofessional communicative process hampers interaction and information sharing for shared decisions that allow the safe continuity of care.
\end{abstract}

\section{DESCRIPTORS}

Communicattion; Emergency Service, Hospital; Patient Care Team; Interprofessional Relations; Patient Safety. 


\section{INTRODUÇÃO}

No Sistema Único de Saúde brasileiro, organizado por meio do estabelecimento das Redes de Atenção à Saúde, o Serviço Hospitalar de Emergência é um dos principais componentes da rede de urgência e emergência e porta de entrada para a alta complexidade ${ }^{(1)}$. Fatores internos e externos a esta unidade, como alternativa para atendimento e possibilidade de acesso à assistência de maior resolutividade pela população, superlotação, dificuldade de atuação integrada da equipe em um ambiente de incertezas, elevada rotatividade, gravidade de pacientes, multiplicidade de tarefas ocasionando sobrecarga profissional, dentre outros ${ }^{(2-3)}$, apresentam-se como dificuldades que podem provocar distorções na comunicação estabelecida entre os profissionais, interferindo em sua efetividade, além de ocasionar fragmentação do cuidado em saúde.

A comunicação interprofissional (CIP) pode ser compreendida como a capacidade de comunicação efetiva entre pessoas, especialmente de diferentes profissões, de maneira colaborativa ${ }^{(4)}$. Melhorar a comunicação entre os profissionais de saúde envolvidos no cuidado é a segunda meta internacional da segurança do paciente, estabelecida pela Joint Commission International, em parceria com a Organização Mundial da Saúde ${ }^{(5)}$.

A construção do diálogo em um ambiente interprofissional permite a criação de um relacionamento com integração entre os envolvidos que possibilita o reconhecimento do trabalho do outro e suas especificidades, sendo determinante primordial para o alcance da comunicação efetiva entre os profissionais, favorecendo um ambiente de cuidado mais seguro e humanizado ${ }^{(6-7)}$.

Destaca-se que a CIP é uma importante ferramenta que deve ser utilizada desde a chegada do paciente à unidade de emergência (UE) por meio do sistema do Acolhimento com Classificação de Risco (ACCR). Esse sistema permite $o$ atendimento horizontal dos pacientes empregando-se fluxogramas e protocolos adequados recomendados ${ }^{(8)}$. Pacientes, quando são acolhidos e classificados em risco vermelho, requerem encaminhamento imediato para a sala vermelha. Entre esses dois espaços, a comunicação entre os profissionais deve permitir a continuidade do atendimento de maneira segura, minimizando os erros e melhorando a qualidade assistencial.

A produção científica nacional e internacional sobre CIP destaca aspectos que abordam os limites nessa comunicação, a exemplo da ausência de rotinas regulares e planejadas de comunicação entre profissionais de categorias distintas e do uso de ferramentas padronizadas, fragilidade na interação e no uso do diálogo que favoreça o trabalho em equipe, a ambiência desfavorável, dentre outros ${ }^{(2-3,6,9)}$. Porém, não foram identificados estudos que mapeiam os fatores internos e externos que interferem na comunicação, o que confere relevância e inovação ao estudo proposto, que considera a temática na perspectiva da interprofissionalidade em cenário hospitalar de emergência.
$\mathrm{Na}$ prática interprofissional colaborativa, as diferentes categorias profissionais devem trabalhar juntas para obtenção de resultados positivos na assistência à saúde ${ }^{(10)}$. Acredita-se que uma CIP efetiva favorece o trabalho em equipe, o cuidado contínuo ao paciente, minimiza a ocorrência de riscos, de efeitos adversos e contribui para a segurança do paciente $e^{(6-7,10-11)}$. Neste sentido, este estudo justifica-se por contribuir com discussões que auxiliam na compreensão de fatores que interferem na efetividade do processo comunicacional entre profissionais de categorias distintas, em um ambiente crítico como o da unidade de emergência, possibilitando desencadear ações que contribuam para o planejamento da comunicação.

Considerando que a comunicação está entre as seis competências necessárias para uma prática interprofissional colaborativa efetiva ${ }^{(11)}$, levantou-se a seguinte questão de pesquisa: Como fatores internos e externos em uma unidade de emergência interferem na prática comunicativa interprofissional?

Assim, este estudo tem como objetivo mapear fatores internos e externos em uma unidade de emergência que interferem na prática comunicativa interprofissional.

\section{MÉTODO}

\section{Desenho do ESTUdo}

Estudo de caso único ${ }^{(12)}$, descritivo, qualitativo que segue as recomendações dos Critérios Consolidados para Relatos de Pesquisa Qualitativa (COREQ) ${ }^{(13)}$.

\section{Participantes}

Participaram 22 profissionais da equipe de saúde, sendo sete médicos, dez enfermeiros e cinco técnicas de enfermagem. $\mathrm{O}$ interesse foi analisar o fenômeno entre os profissionais atuantes no ACCR e na sala vermelha, espaços percorridos pelo paciente em situação de emergência, seja por demanda espontânea ou regulados.

\section{Cenário}

Unidade de emergência de um hospital geral público, de grande porte, alta complexidade, terciário, no Estado da Bahia. A unidade de emergência utiliza a Classificação de risco pelo protocolo de Manchester visando a priorizar o atendimento por níveis de gravidade ${ }^{(8)}$.

\section{CRITÉRIOS de SELEÇÃo}

Adotou-se como critérios de inclusão: ser profissional da equipe de saúde fixo da unidade, atuante no cuidado direto ao paciente na unidade de emergência, durante o período de coleta dos dados. Não houve critério de exclusão.

\section{DeFINIÇÃo DA AMOSTRA}

A seleção foi realizada por meio de amostragem não probabilística intencional por bola de neve ${ }^{(14)}$. A primeira participante foi indicada pela diretoria do hospital, enfermeira 
assistencial no ACCR e na sala vermelha. Essa, por sua vez, indicou outras pessoas a serem entrevistadas e assim por diante, não havendo recusas, com prosseguimento para a coleta até a obtenção de cada vez menos informações a cada entrevista.

\section{Coleta de dados}

O uso de múltiplas fontes de evidência possibilita desenvolver linhas convergentes de investigação em um estudo de caso $^{(12)}$. As fontes de evidência utilizadas neste estudo foram: a observação participante, a entrevista semiestruturada e a análise documental. A coleta de dados ocorreu em três momentos distintos e complementares e as informações coletadas foram trianguladas, permitindo a convergência de evidências que ajudaram a reforçar a validade do constructo ${ }^{(12)}$.

No primeiro momento, a observação participante, realizada de maneira livre, durante 30 dias não consecutivos, somando 50 horas, em turnos e horários variados, permitiu a inserção da pesquisadora no cotidiano da unidade de emergência. Foi adotado como ponto de partida dessa etapa o questionamento: como ocorre o fluxo de comunicação sobre o paciente entre os profissionais que atuam no ACCR e entre esses e os que atuam na sala vermelha? A observação foi mantida até serem identificados indícios de saturação, ou seja, a obtenção de cada vez menos informações novas a cada sessão. Os dados observados foram registrados no diário de campo e, posteriormente, transcritos na íntegra.

No segundo momento, realizou-se a entrevista semiestruturada, em local reservado na instituição, seguindo roteiro, contendo dados de caracterização e questões norteadoras relativas à importância da comunicação: como essa ocorre, fatores que interferem na comunicação e sugestões para sua melhoria. Os dados da entrevista, que teve duração entre 15 e 60 minutos, foram gravados e transcritos na íntegra.

No terceiro momento, dados secundários foram obtidos por meio dos instrumentos escritos utilizados pelos profissionais do ACCR (ficha de avaliação do paciente) e da sala vermelha (prontuário), onde houvesse informações relacionadas aos pacientes, e dos relatórios produzidos pelo Departamento de Qualidade e Vigilância em Saúde da instituição. A partir da leitura inicial dos documentos selecionados, foi realizada uma triagem dos materiais e, posteriormente, realizada a leitura minuciosa que permitiu conhecer como as informações eram registradas, e se a comunicação entre a equipe de saúde através destes documentos possibilitava a continuidade do cuidado prestado ao paciente.

A triangulação de dados possibilitou a apreensão de informações acerca do objeto de estudo em diferentes tipos de conhecimentos e níveis de profundidade. Assim, os dados obtidos nos registros do diário de campo, nas entrevistas com os participantes da pesquisa e nos documentos possibilitaram conhecer o fluxo da comunicação, como essa ocorre, qual a sua importância para os profissionais médicos, enfermeiros e técnicos de enfermagem; quais fatores interferem, e se as informações registradas eram complementares e contribuíam para a condução coordenada do cuidado ao paciente.

\section{ANÁLISE E TRATAMENTO DOS DADOS}

As informações foram analisadas por meio da técnica de análise temática de Bardin ${ }^{(15)}$. Primeiramente, na etapa de pré-análise, os dados foram organizados gerando o corpus da pesquisa. Em seguida, foi iniciada a exploração do material através de leitura flutuante e exaustiva das transcrições, sendo apreendidas ideias centrais e estruturas de relevância do estudo, possibilitando as operações de codificação e categorização do material, com base na questão de investigação e a fase de tratamento dos resultados.

A partir dos dados produzidos e com o uso da Matriz Swot ${ }^{(16)}$, mapearam-se os fatores internos e externos que interferem na prática comunicativa na unidade do estudo. A ferramenta de gestão matriz de análise SWOT, sigla derivada da língua inglesa, que se refere a quatro componentes: Strengths (Forças), Weaknesses (Fraquezas), Opportunities (Oportunidades) e Threats (Ameaças), permite analisar os fatores internos e externos ligados a uma organização, e mapear informações e situações gerais, a fim de auxiliar no planejamento estratégico e no processo de tomada de decisão ${ }^{(16)}$.

Os dados foram organizados a partir de categorias definidas a priori, tendo como base os fundamentos da matriz SWOT: pontos fortes e fracos, oportunidades e ameaças na unidade de emergência, além de inferências analíticas relacionadas a cada um desses pontos, conforme organização da análise temática.

\section{Aspectos ÉTICOS}

A pesquisa respeitou os princípios éticos da Resolução n ${ }^{\circ}$ 466/2012 do Conselho Nacional de Saúde e foi aprovada pelo Comitê de Ética em Pesquisa com Seres Humanos do Hospital Geral lócus, no ano de 2019, sob parecer n. 3.159.649. Para preservar o anonimato dos participantes, foi utilizada uma codificação nominal, substituindo nomes por letras conforme a categoria profissional e uma codificação numérica para indicar o número da entrevista correspondente.

\section{RESULTADOS}

Dos 22 profissionais participantes do estudo, houve predominância do sexo feminino (12), a idade variou de 27 a 54 anos, com média de 30,7 anos. O tempo de formação variou de 1 a 25 anos e o tempo de atuação na unidade de emergência de 1 mês a 8 anos.

A identificação dos pontos fortes, pontos fracos, oportunidades e ameaças da comunicação na unidade de emergência (Quadro 1) a partir da matriz de Análise SWOT permitiu conhecer fatores internos e externos que interferem nas práticas comunicativas produzidas nas interações dos profissionais da equipe de saúde. 
Quadro 1 - Matriz SWOT dos fatores internos e externos que interferem na comunicação interprofissional na UE - Salvador, Bahia, 2019.

\begin{tabular}{|c|c|}
\hline $\begin{array}{l}\text { PONTOS FORTES } \\
\text {-Equipe compreende a comunicação como medida de segurança; } \\
\text {-Núcleo interno de segurança; } \\
\text {-Assessoria de comunicação. }\end{array}$ & $\begin{array}{l}\text { OPORTUNIDADES } \\
\text {-Investimentos do setor público para reforma na Unidade de Emergência } \\
\text { com ampliação do número de leitos, além de investimentos para reforma } \\
\text { no Centro Cirúrgico e leitos de enfermaria. }\end{array}$ \\
\hline $\begin{array}{l}\text { PONTOS FRACOS } \\
\text {-Superlotação; } \\
\text {-Atendimento por ordem de chegada em situação de superlotação; } \\
\text {-Sobrecarga; } \\
\text {-Ausência na padronização de condutas assistenciais; } \\
\text {-Profissionais sem experiência; } \\
\text {-Déficit no relacionamento interprofissional; } \\
\text {-Falhas na comunicação verbal oral e escrita. }\end{array}$ & $\begin{array}{l}\text { AMEAÇAS } \\
\text {-Interrupção no funcionamento da Unidade de Pronto Atendimento situada } \\
\text { em frente ao hospital lócus. } \\
\text {-Ausência de treinamento no uso do Protocolo de Manchester. }\end{array}$ \\
\hline
\end{tabular}

Pontos fortes da unidade de emergência: Fatores internos que interferem positivamente na comunicação dos profissionais

A comunicação é reconhecida pelos profissionais como medida de segurança, e as ferramentas comunicacionais utilizadas, como a comunicação oral pela passagem de plantão, e a comunicação escrita por meio da ficha de avaliação do paciente no ACCR e do prontuário, utilizado quando há necessidade de encaminhamento para a sala vermelha, para serem seguras, devem ser objetivas, com linguagem única, e capazes de compartilhar as condutas instituídas, dando continuidade às mesmas.

Quando você recebe todas as informações com coerência, você oferece uma melhor assistência ao paciente, de maneira segura e sem colocar a nossa vida e a de outras pessoas em risco (TE10).

A comunicação adequada permite a continuidade do cuidado. Vocêpercebe a diferença de um paciente que chega direto da porta para a sala vermelha e o que passa pelo ACCR. Conseguimos ter melhores informações quando ele vem do ACCR (E6).

[...] nosso objetivo é deixar o prontuário bem escrito, para que o colega entenda o que a gente pensou, o que foi feito, e o que se espera encontrar, para dar continuidade (M11).

Para apoiar as ações de segurança, o hospital possui um Núcleo Interno de Segurança que, em parceria com a sua assessoria de comunicação, realiza campanhas pontuais para sensibilizar os profissionais acerca das medidas de segurança a serem instituídas diariamente, destacando a importância da comunicação interprofissional para a condução das ações em benefício do paciente.

Pontos fracos da unidade de emergência: Fatores internos que interferem negativamente na comunicação dos profissionais

Fatores internos fragilizam o processo comunicativo interprofissional, com destaque para a superlotação da unidade que ocasionava atendimentos por ordem de chegada, e não por prioridade, com demanda elevada de atendimentos não urgentes, promovendo sobrecarga para os profissionais e fragilizando a comunicação entre eles.

[...] a demanda e o fuxo são grandes, tudo é muito rápido e faz com que as pessoas não parem para ouvir direito as informações transmitidas (E15).

Você lida diariamente com situaçôes naturalmente estressantes e a falta de infraestrutura, a superlotação, a falta de recursos humanos adequados, interferem na comunicação entre os profissionais (M16).
A comunicação na sala vermelha é deficiente, pois está sempre cheia de pacientes e profissionais. Muitas vezes, o paciente do ACCR dá entrada e nẫo somos comunicados. Quando menos se espera, está aqui dentro, não sabemos o nome e nem porque ele veio (TE18).

A ausência na padronização de condutas assistenciais, falhas na passagem de plantão e profissionais sem experiência fragmentavam o compartilhamento de informações entre os profissionais, ocasionando uma comunicação de maneira pontual, dificultando a continuidade das ações para promoção do cuidado seguro.

A ausência de padronização na passagem de informações devido à falta de organização da unidade faz com que informaçôes sejam veiculadas de maneira incompleta (M11).

A passagem de plantão é frágil e com muitas informaçôes perdidas, levando a perda de tempo. Se as informações fossem adequadas conseguiria agilizar e otimizar o cuidado do paciente (E5).

A inexperiência de muitos profissionais com a ausência de padronização de condutas faz com que as decisóes para o cuidado sofram mudanças constantes. Isso cria conflito entre as equipes, pois os profissionais não sentem segurança nas informaçôes transmitidas (E15).

A comunicação verbal oral ocorria sem um protocolo padronizado, contribuindo para ausência de uniformidade, com pouca interação interprofissional, e entre uma mesma categoria profissional. Ademais, sofria interferências constantes devido a ruídos de aparelhos e monitores, conversas paralelas, interrupções por outros profissionais que não faziam parte da equipe, quebrando o raciocínio das pessoas envolvidas e dificultando o compartilhamento das informações acerca do cuidado.

Ao analisar a ficha de atendimento do usuário preenchida por médicos e enfermeiros no ACCR, identificou-se que nem todos os profissionais preenchiam-na de maneira completa. Apesar da existência de impressos informatizados, as fichas de evolução médica e de enfermagem eram preenchidas manualmente, comprometendo a legibilidade. Também, foi identificada repetitividade em vez de complementaridade das informações registradas por médicos, enfermeiros e técnicos de enfermagem.

O déficit no relacionamento interprofissional foi outro ponto fraco identificado e sofria influência da hierarquia estabelecida. Observou-se que o relacionamento entre os 
profissionais envolvidos no atendimento dos pacientes que davam entrada no ACCR e eram encaminhados para a sala vermelha ocorria apenas entre profissionais de uma mesma categoria profissional.Já o relacionamento estabelecido pela enfermeira do ACCR com o médico da sala vermelha era praticamente inexistente, e poucos profissionais médicos e enfermeiros consideravam interagir com os técnicos de enfermagem, limitando a comunicação para solicitar a realização de procedimentos.

Os médicos do ACCR entram na sala vermelha e se dirigem aos médicos. A enfermeira passa as informações para a enfermeira, $e$ ela nos informa o que fazer pelo paciente (TE13).

A comunicação do médico com a equipe de enfermagem é mais escrita do que verbal. A comunicação verbal depende da relação que o médico tem com a gente. Se ele nos conhece, nos procura para esclarecer alguma dúvida, ou discutir algo em relação ao paciente (E3).

A falha na transmissão de informações entre o enfermeiro, o técnico de enfermagem e o médico interfere no cuidado. O médico deveria passar as informaçóes de maneira clara, discutir o que está planejando, para que todos fiquem sabendo (M7).

Oportunidades: Fatores do ambiente externo que interferem na comunicação dos profissionais.

Os investimentos do setor público para reforma na UE do HG, com ampliação do número de leitos, e reforma no Centro Cirúrgico e dos leitos de enfermaria, são oportunidades importantes para a melhoria do trabalho e, consequentemente, da comunicação interprofissional. Porém, essas reformas ainda não haviam sido iniciadas na instituição, sendo necessário verificar condições e viabilidade para utilização dessas oportunidades.

Ameaças: Fatores do ambiente externo que impedem o desenvolvimento organizacional efetivo, interferindo na comunicação interprofissional.

As interrupções periódicas no funcionamento da Unidade de Pronto Atendimento, próxima ao HG, por superlotação, ocasionava também superlotação da UE, sendo ameaça para o estabelecimento da CIP.

Foi pontuado que a ausência de capacitação dos profissionais do ACCR, para classificação de risco, contribui com falhas na CIP sobre o paciente nesses espaços, e entre eles. Embora a capacitação seja oferecida pela Secretaria Municipal de Saúde do município, esta não ocorre há mais de três anos, conforme informação dos gestores.

[...] a ausência de treinamentos faz com que a equipe do ACCR falhe. Se chega o paciente grave e não forem passadas as informações corretas na sala vermelha, há o risco de morte (E1).

[...] a ausência de treinamento impacta no momento que o enfermeiro vai transferir informações sobre o paciente para a sala vermelha. Ele não capta a queixa correta, e preenche o fluxograma sem ser condizente, passando informações inapropriadas (E15).

\section{DISCUSSÃO}

As informações produzidas relacionadas ao estado de saúde e necessidades das pessoas atendidas na unidade de emergência requerem ser disponibilizadas por meio de uma rede efetiva de compartilhamento interprofissional, contribuindo no processo de trabalho e na produção do cuidado. Para os participantes, a CIP no compartilhamento de informações sobre o paciente é uma importante medida de segurança, devendo ser objetiva para o planejamento e a execução de ações contínuas.

Porém, identificou-se fragilidade comunicativa entre os profissionais da unidade de emergência lócus, em consequência de fatores como superlotação, sobrecarga de trabalho, falta de recursos materiais e humanos, inexperiência de profissionais e ausência na padronização de condutas. Tais fatores refletem em um processo de trabalho que fragmenta o cuidado, repercutindo na comunicação, e trazendo, como consequência, a desarticulação da assistência e a insatisfação entre profissionais e pacientes ${ }^{(17)}$.A superlotação, por exemplo, é considerada um fenômeno mundial, que interfere na qualidade da prestação do serviço, na dinâmica de trabalho, e na segurança do cuidado ${ }^{(17-18)}$.

Nesse contexto, o compartilhamento de informações verbais sobre o paciente, realizada por profissionais de categorias distintas, com interferências constantes, associada à ausência de uma ferramenta de comunicação padronizada, promove perda na uniformidade das informações. A comunicação escrita, composta por informações incompletas, repetitivas e pouco legíveis, dificulta a compreensão sobre o cuidado e o plano terapêutico.

Falhas na comunicação com quantidade excessiva ou reduzida de informações, e as informações inconsistentes, são fatores que interferem na $\operatorname{CIP}^{(9)}$ e na continuidade da assistência segura. É desafiador identificar métodos e adotar estratégias que diminuam a deterioração da informação, com a perda de dados clínicos importantes durante a comunicação em setores críticos ${ }^{(3)}$. Logo, protocolos e procedimentos organizacionais devem ser instituídos para garantir que todos os membros da equipe estejam presentes e todas as informações disponíveis ${ }^{(19)}$.

Um estudo prospectivo pré e pós intervenção objetivando determinar o impacto de um programa de melhoria na comunicação verbal entre os enfermeiros em uma unidade de terapia intensiva evidenciou impacto positivo na comunicação, incluindo avaliação da gravidade da doença (37\% pré-intervenção versus $67 \%$ pós-intervenção), diminuição da frequência de interrupções, e ausência de impacto negativo no fluxo de trabalho da enfermagem. Assim, revelou a importância de programas de treinamento padronizados, com foco na melhora da CIP para redução de $\operatorname{erros}^{(9)}$.

O programa de melhoria do estudo acima citado foi realizado por meio de treinamento educacional sobre as melhores práticas de comunicação verbal, implementação mnemônica de transferência verbal e materiais visuais. Os enfermeiros foram instruídos quanto a elementos importantes a serem compartilhados, o uso de um formato padronizado que inicie com informações gerais e siga com informações específicas, e sobre a importância de fornecer informações atualizadas e relevantes.

Uma ferramenta eficaz para uso na unidade de emergência é o SBAR. Composto pelo acrônimo situation (situação), background (antecedentes), assessment (avaliação) and 
recommendation (recomendações), é um método estruturado para padronizar a troca de informações e a sequência de atuação dos profissionais de saúde, contribuindo para a segurança do paciente ${ }^{(20-21)}$. Permite, de uma maneira fácil e focada, definir expectativas para o que será comunicado entre os membros da equipe, possibilitando o desenvolvimento do trabalho interprofissional. Estudo de métodos mistos que consistiu em avaliar o impacto da utilização do SBAR na qualidade das transferências na admissão em um departamento de emergência revelou melhora na qualidade da comunicação verbal entre os profissionais, contribuindo para delinear a responsabilidade no cuidado e para agilizar o fluxo ${ }^{(22)}$.

A comunicação escrita na unidade do estudo ocorria, predominantemente, por meio do prontuário. Esse é um documento legal utilizado para o registro diário de informações na unidade hospitalar, e deve transmitir informações capazes de promover melhorias na assistência prestada ao paciente ${ }^{(23)}$. Porém, devido à ilegibilidade, é necessária a informatização do prontuário, algo ainda incipiente na unidade estudada. A comunicação escrita deve estar alinhada com o sistema informatizado, possibilitando que os dados sejam registrados de maneira consistente e clara, com informações cruciais destacadas e de fácil visualização ${ }^{(19)}$. "A eficácia no desdobramento de informações escritas e orais, para os diversos profissionais, promove autonomia para as decisões e mais envolvimento dos níveis assistenciais com ações de melhoria da qualidade da assistência ${ }^{(24)}$.

Para isso, o aspecto relacional é importante. Entretanto, evidenciou-se no estudo que esse foi um ponto fraco na unidade, associado a questões hierárquicas, ausência de vínculo entre os profissionais, principalmente entre categorias profissionais distintas. Estudo que buscou compreender a visão dos profissionais de Enfermagem de um pronto-socorro sobre a transferência de cuidado de pacientes revelou a falta de diálogo, a omissão de informações e a desigualdade na valoração dos distintos trabalhos como dificultadores para a construção de relações interprofissionais horizontais, essas últimas capazes de contribuir para o estabelecimento da comunicação efetiva e o trabalho em equipe ${ }^{(3)}$.

Um aspecto que despertou atenção, tanto nas falas como na observação, foi a fragilidade da comunicação dos profissionais enfermeiros e médicos com os técnicos de enfermagem. O compartilhamento de informações ocorria, usualmente, entre os médicos e os enfermeiros, e o técnico de enfermagem mantinha-se em silêncio ao lado destes profissionais, executando tarefas delegadas. Esse achado assemelha-se àquele encontrado em outro estudo que destacou a não valorização e o sentimento de inferioridade como fatores que dificultavam a comunicação desse profissional com os demais da equipe. Observaram-se também dificuldades de trabalho em equipe envolvendo profissionais de enfermagem, que atuam com foco na execução de tarefas e procedimentos, e falta de cooperação e distanciamento com a equipe médica ${ }^{(3)}$.

A persistência de práticas hierarquizadas e desiguais entre as diferentes categorias profissionais na área de saúde, os silos (grupos de profissionais que atuam de maneira isolada), o poder, os conflitos no trabalho e a dificuldade em compreender o papel do outro favorecem a atuação em paralelo dos profissionais, em detrimento do trabalho em equipe, influenciando o modo como a comunicação interprofissional é estabelecida, podendo acarretar falhas na comunicação, com risco para os pacientes ${ }^{(19,24)}$.

A dificuldade no estabelecimento do trabalho em equipe no cotidiano das práticas assistenciais em saúde sofre influência da hegemonia do modelo tradicional biomédico e fragmentado, um dos grandes desafios do sistema de saúde brasileiro na atualidade ${ }^{(25)}$. Essa realidade opõe-se ao entendimento de equipe de profissionais que deveriam integrar os diferentes saberes por meio da colaboração interprofissional, reconhecendo sua interdependência "pautada na prática comunicativa intersubjetiva entre os trabalhadores das equipes, e destes com os usuários e famílias" ${ }^{(7)}$, com vistas a propiciar uma atenção mais qualificada, centrada no usuário e em suas necessidades de saúde ${ }^{(6-7)}$.

O desenvolvimento do trabalho em equipe na situação de emergência deve considerar a aplicação do conhecimento técnico-científico, esse de caráter instrumental, em comunhão com o estabelecimento da comunicação entre os profissionais de diferentes categorias que atuam juntos, possibilitando o compartilhamento de informações acerca da condição de saúde do usuário e definição de prioridades para o cuidado. Sob esta perspectiva, a prática compartilhada com profissionais de outras áreas, centrada na atenção às necessidades de saúde do usuário, por meio da construção de relações simétricas e lineares, contribui para o desenvolvimento da $\mathrm{PIC}^{(7)}$, melhora da qualidade da atenção à saúde possibilitando a tranversalização das ações para além do atendimento na situação aguda enfrentada pelo paciente.

A utilização de estratégias educacionais, psicológicas e organizacionais possibilita superar as barreiras de comunicação da equipe interprofissional, por meio da aplicação de métodos estruturados de aprendizagem; capacitação da equipe interprofissional; treinamentos de habilidades de comunicação por meio da simulação; redefinição da "equipe" de profissionais, com criação de equipes democráticas, favorecendo o sentimento de pertencimento em cada membro, fortalecendo o sentimento de valorização e de confiança; além de protocolos e procedimentos organizacionais ${ }^{(19)}$.

Um estudo que objetivou analisar estratégias adotadas para melhorar a comunicação interna em um hospital geral destacou um projeto cujo foco era a continuidade da prática profissional qualificada durante 24 horas, por meio da uniformização da comunicação. Para os autores, a comunicação efetiva, aliada ao engajamento e envolvimento dos profissionais sobre suas ações, possibilitou a construção de uma cultura única referente a qualidade e eficácia de informações estratégicas, promovendo autonomia para a tomada de decisões dos profissionais ${ }^{(24)}$.

Com relação às ameaças, os participantes do projeto em tela destacaram a deficiência da rede loco regional, que interferiu no planejamento da unidade, contribuindo para a superlotação e a consequente deficiência na CIP. Evidência aponta que a desarticulação dos serviços na rede de atenção às urgências fragiliza o acesso qualificado, e acarreta problemas na segurança e privacidade do paciente ${ }^{(26)}$, corroborando com os achados deste estudo. 
Outra ameaça a ser destacada é a ausência de treinamento dos profissionais que atuam no ACCR quanto ao uso do protocolo de Manchester. A falta de capacitação periódica para a atualização desses profissionais contradiz o preconizado pelos protocolos de classificação de risco ${ }^{(26)}$. É importante salientar que o ACCR é uma área que objetiva oferecer atendimento imediato. A ausência ou escassez de informações decorrente da inexperiência profissional torna-se impeditivo para a conduta direcionada, rápida e uniforme.

$\mathrm{O}$ estudo, embora realizado em uma única UE, reflete pontos fortes, pontos fracos, ameaças e oportunidades comuns a muitas unidades de emergência nacionais e internacionais, principalmente no que se refere à superlotação ${ }^{(17-18)}$, relação entre a equipe ${ }^{(2-3)}$ e investimento no setor público. Entretanto, apresentou limitação, visto que o cenário apontado se refere a uma unidade pública de saúde, que traz as suas particularidades. Portanto, os resultados devem ser confrontados em novos estudos, a fim de ampliar a compreensão sobre a problemática em questão.

Este estudo irá contribui com a discussão da comunicação no serviço de emergência, dando visibilidade a problemas complexos da equipe de saúde em organizações hospitalares. Os resultados podem subsidiar a construção de estratégias efetivas de CIP para que esta faça parte da cultura interna organizacional.

\section{CONCLUSÃO}

Fatores internos e externos à unidade de emergência interferem tanto positiva quanto negativamente na comunicação entre os profissionais. Esses compreendem a importância da comunicação como medida de segurança, utilizam a passagem de plantão e os registros escritos para compartilhar informações. Porém, a superlotação, a sobrecarga de trabalho, a ausência de padronização de condutas, profissionais sem experiência, e déficit no relacionamento entre os que atuam em categorias distintas contribuem para que a interação e o processo comunicativo interprofissional se torne fragilizado, e dificulta o compartilhamento de informações para a tomada de decisão compartilhada.

Assim, é necessária a superação dos aspectos que nela interferem, com mudança no paradigma assistencial do biomédico para o interprofissional, permitindo o fortalecimento da assistência centrada no paciente e não nos profissionais. É necessário que os profissionais reconheçam a importância do trabalho em equipe, estabeleçam relações de trabalho mais dialógicas com ações de cuidado integradas, mudem seus comportamentos relacionados à prática comunicativa interprofissional e, em parceria com a gestão organizacional, planejem e implementem ações que contribuam para a comunicação efetiva, melhorando ferramentas já utilizadas.

\section{RESUMO}

Objetivo: Mapear fatores internos e externos em uma unidade de emergência que interferem na prática comunicativa interprofissional. Método: Estudo de caso único realizado na unidade de emergência de um hospital geral. Os dados foram coletados por meio de observação participante, análise documental e entrevista semiestruturada, triangulados e submetidos à análise temática, a partir de categorias definidas a priori, baseadas na matriz SWOT. Resultados: Participaram 22 profissionais de saúde. Quanto aos pontos fortes e oportunidades, constatou-se que os profissionais compreendem a importância da comunicação como medida de segurança, utilizam a passagem de plantão e a comunicação escrita para compartilhar informações. Porém, a superlotação, a sobrecarga de trabalho, a ausência de padronização de condutas, a inexperiência de profissionais e o déficit no relacionamento interprofissional são fatores dificultadores de uma comunicação efetiva. Conclusão: $O$ processo comunicativo interprofissional fragilizado dificulta a interação e o compartilhamento de informações para a tomada de decisões compartilhadas que possibilite a continuidade do cuidado de maneira segura.

\section{DESCRITORES}

Comunicação; Serviço Hospitalar de Emergência; Equipe de Assistência ao Paciente; Relações Interprofissionais; Segurança do Paciente.

\section{RESUMEN}

Objetivo: Mapear factores internos y externos en una unidad de urgencias que interfieren en la práctica comunicativa interprofesional. Método: Estudio de un único caso realizado en la unidad de urgencias de un hospital. Los datos fueron colectados por observación participante, análisis documental y entrevista semi estructurada, triangulados y sometidos al análisis temático, a partir de categorías definidas a priori, basadas en la matriz SWOT. Resultados: Participaron 22 profesionales de salud. Se constató que los profesionales comprenden la importancia de la comunicación como medida de seguridad, utilizan la entrega de turno y la comunicación escrita para compartir informaciones. Pero, los hospitales muy concurridos, el exceso de trabajo, la ausencia de una estandarización de conductas, la inexperiencia profesional y el déficit en las relaciones interprofesionales son obstáculos para que la comunicación sea efectiva. Conclusión: el proceso comunicativo interprofesional debilitado dificulta la interacción y la división de informaciones para la toma de decisiones compartidas que permita la continuación del cuidado de manera segura.

\section{DESCRIPTORES}

Comunicación; Servicio de Urgencia en Hospital; Grupo de Atención al Paciente; Relaciones Interprofesionales; Seguridad del Paciente.

\section{REFERÊNCIAS}

1. O'Dwyer G, Konder MT, Reciputti LP, Macedo C, Lopes MG. [Implementation of the Mobile Emergency Medical Service in Brazil: action strategies and structural dimension]. Cad Saude Publica. 2017;33(7):e00043716. Portuguese. https://doi.org/10.1590/0102-311x00043716

2. Redley B, Botti M, Wood B, Bucknall T. Interprofessional communication supporting clinical handover in emergency departments: an observation study. Australas Emerg Nurs J. 2017;20(3):122-30. https://doi.org/10.1016/j.aenj.2017.05.003

3. Alves M, Melo L. Handoff of care in the perspective of the nursing professionals of an emergency unit. Rev Min Enferm. 2019;23:e-1194. https://doi.org/10.5935/1415-2762.20190042 
4. Canadian Interprofessional Health Collaborative. A National Interprofessional Competency Framework. College of Health Disciplines University of British Columbia [Internet]. Vancouver: Canadian Interprofessional Health Collaborative ; 2010 [cited 2019 Sep 9]. Available from: https://www.academia.edu/25496437/A_National_Interprofessional_Competency_Framework

5. Brasil. Ministério da Saúde. Documento de referência para o Programa Nacional de Segurança do Paciente [Internet]. Rio de Janeiro: Instituto de Comunicação e Informação Científica e Tecnológica em Saúde; 2014 [cited 2018 Sep 18]. Available from: https://proqualis. net/livro/documento-de-refer\%C3\%AAncia-para-o-programa-nacional-de- seguran\%C3\%A7a-do-paciente

6. Olino L, Gonçalves AC, Strada JK, Vieira LB, Machado ML, Molina KL, et al. Effective communication for patient safety: transfer note and Modified Early Warning Score. Rev Gaúcha Enferm. 2019;40(esp):e20180341. https://doi.org/10.1590/1983-1447.2019.20180341

7. Peduzzi M, Agreli HL, Silva JA, Souza HS. [Teamwork: revisiting the concept and its developments in inter-professional work]. Trab Educ Saúde. 2020;18(suppl. 1):e0024678. Portuguese. https://doi.org/10.1590/1981-7746-sol00246

8. Costa MA, Versa GL, Bellucci Júnior JA, Inoue KC, Sales CA, Matsuda LM. Admittance of risk-classified cases: assessment of hospital emergency services. Esc Anna Nery. 2015;19(3):491-7. https://doi.org/10.5935/1414-8145.20150065

9. Starmer AJ, Schnock KO, Lyons A, Hehn RS, Graham DA, Keohane C, et al. Effects of the I-PASS Nursing Handoff Bundle on communication quality and workflow. BMJ Qual Saf. 2017;26(12):949-57. https://doi.org/10.1136/bmjqs-2016-006224

10. Reeves S, Pelone F, Harrison R, Goldman J, Zwarenstein M. Interprofessional collaboration to improve professional practice and healthcare outcomes. Cochrane Database Syst Rev. 2017;6:CD000072. https://doi.org/10.1002/14651858.CD000072.pub3

11. Previato GF, Baldissera VD. Communication in the dialogical perspective of collaborative interprofessional practice in Primary Health Care. Interface (Botucatu). 2018;22(Suppl.2):1535-47. https://doi.org/10.1590/1807-57622017.0647

12. Yin RK. Estudo de caso: planejamento e métodos. 5th ed. Porto Alegre: Bookman; 2015.

13. Tong A, Sainsbury P, Craig J. Consolidated criteria for reporting qualitative research (COREQ): a 32-item checklist for interviews and focus groups. Int J Qual Health Care. 2007;19(6):349-57. https://doi.org/10.1093/intqhc/mzm042

14. Vinuto J. Snowball sampling in qualitative research: an open debate. Temáticas. 2014;22(44):203-20. https://doi.org/10.20396/tematicas.v22i44.10977

15. Bardin L. Análise de conteúdo. São Paulo: Edições 70; 2016.

16. Gurel E. TAT M. SWOT analysis: a theoretical review. J Int Soc Res. 2017;10(51):994-1006. https://doi.org/10.17719/jisr.2017.1832

17. Dal Pai D, Lautert L. Suffering in the nursing work: reflexes of "empty speech" at the embracement with risk classification. Esc Anna Nery. 2011;15(3):524-30. https://doi.org/10.1590/S1414-81452011000300012

18. Yarmohammadian MH, Rezaei F, Haghshenas A, Tavakoli N. Overcrowding in emergency departments: A review of strategies to decrease future challenges. J Res Med Sci. 2017;22(1):23. https://doi.org/10.4103/1735-1995.200277

19. Weller J, Boyd M, Cumin D. Teams, tribes and patient safety: overcoming barriers to effective teamwork in healthcare. Postgrad Med J. 2014;90(1061):149-54. https://doi.org/10.1136/postgradmedj-2012-131168

20. Institute for Healthcare Improvement. SBAR technique for communication: a situational briefing model. 2015 [cited 2018 May 21 ]. Available from: http://www.ihi.org/resources/Pages/Tools/SBARTechniqueforCommunicationASituationalBriefingModel.aspx

21. Müller M, Jürgens J, Redaèlli M, Klingberg K, Hautz WE, Stock S. Impact of the communication and patient hand-off tool SBAR on patient safety: a systematic review. BMJ Open. 2018;8(8):e022202. https://doi.org/10.1136/bmjopen-2018-022202

22. Smith CJ, Buzalko RJ, Anderson N, Michalski J, Warchol J, Ducey S, et al. Evaluation of a novel handoff communication strategy for patients admitted from the emergency department. West J Emerg Med. 2018;19(2):372-9. https://doi.org/10.5811/westjem.2017.9.35121

23. Ferreira LL, Chiavone FB, Bezerril MD, Alves KY, Salvador PT, Santos VE. Analysis of records by nursing technicians and nurses in medical records. Rev Bras Enferm. 2020;73(2):e20180542. https://doi.org/10.1590/0034-7167-2018-0542

24. Freire EM, Silva, VC, Adriane Vieira A, Matos SS, Alves M. Communication as a strategy for hospital accreditation maintenance. Esc Anna Nery. 2019;23(1):e20180224. https://doi.org/10.1590/2177-9465-ean-2018-0224

25. Fertonani HP, Pires DE, Biff D, Scherer MD. Communication as a strategy for hospital accreditation maintenance. Cienc Saúde Coletiva. 2015;20(6):1869-78. https://doi.org/10.1590/1413-81232015206.13272014

26. Duro CL, Lima MA, Weber LA. [Nurses' opinion on risk classification in emergency services]. REME - Rev Min Enferm. $2017 ; 21: e 1062$. Portuguese. https://doi.org/10.5935/1415-2762.20170072 